\title{
Kawasaki Disease and Peripheral Gangrene in Infancy
}

\author{
Iran Malekzadeh, ${ }^{1}$ Vahid Ziaee, ${ }^{2,3,{ }^{*}}$ Taravat Sadrosadat, ${ }^{1}$ Mohammad-Hassan Moardinejad, ${ }^{1,3}$ \\ and Keyhan Sayadpour-Zanjani ${ }^{3}$ \\ ${ }^{1}$ Children's Medical Center, Pediatrics Center of Excellence, Tehran University of Medical Sciences, Tehran, IR Iran \\ ${ }^{2}$ Rheumatology Research Center, Tehran University of Medical Sciences, Tehran, IR Iran \\ 3 Department of Pediatrics, Tehran University of Medical Sciences, Tehran, IR Iran \\ *Corresponding author: Vahid Ziaee, Rheumatology Research Center, Tehran University of Medical Sciences, Tehran, IR Iran. Tel:+9821-66595522, Fax: +9821-66595525, E-mail: ziaee@tums.ac.ir
}

Received 2015 July 5; Revised 2015 October 13; Accepted 2015 October 20

\begin{abstract}
Introduction: Early diagnosis and treatment of Kawasaki disease as the most common cause of acquired heart disease in childhood, may significantly improve the prognosis. Diagnosing infantile Kawasaki (younger than a year) is difficult because of obscure symptoms; at the same time they are at the higher risk of coronary abnormalities.

Case Presentation: We report three infants with prolonged (more than 5 days) fever and peripheral gangrene without any other clinical manifestations of Kawasaki disease. Kawasaki was diagnosed due to dilation of coronary artery and other aortic branches, thrombocytosis, and rising of ESR and CRP. All patients were treated with high dose aspirin, IVIG and pulse therapy with methylprednisolone. Additionally, cytotoxic drugs or infliximab were used for two of them because of severe aneurysms in the aortic branches. All 3 patients received aspirin with anti-platelet aggregation dose and 2 patients heparin as an anti-coagulant agent for longtime. After adequate treatment, peripheral gangrene, arterial dilations and aneurysms improved, but during 12 months follow-up coronary aneurysms did not improve completely. Conclusions: Peripheral gangrene must be regarded as an important sign of infantile Kawasaki disease early treatment of which can prevent severe permanent coronary involvements and sequels.
\end{abstract}

Keywords: Kawasaki Disease, Infantile Polyarthritis, Gangrene, Vasculitis

\section{Introduction}

Kawasaki disease (KD) as a febrile vasculitis is the second common vasculitis of childhood after Henoch-Schönlein Purpura. Its prevalence is 239 cases per 100,000 children under 5 years old in Asian countries vs about 50 per 100,000 in USA (1-3). Eighty percent of affected children are under 4 years old (4) and the peak age of incidence is 9 - 11 months (2). KD is the most common cause of acquired heart disease of infancy and childhood. Without sufficient treatment, it may cause coronary dilation and aneurysm in $25 \%$ of patients $(1,5)$; subsequently stenosis, thrombosis, myocardial infarction and congestive heart failure may happen (6). Early diagnosis and treatment may improve the prognosis significantly. Diagnosing infantile Kawasaki (younger than one year) is difficult because of obscure symptoms (7); at the same time they are at the higher risk of coronary abnormalities (8-11). Other cardiovascular involvements such as pericardial effusion, mitral insufficiency, congestive heart failure, myocardial systolic dysfunction, and systemic vasculitis (iliac, renal, axillary and mesenteric arteries) were reported $(1,12-16)$. Even rare interpretation of peripheral gangrene and amputation had been detected due to peripheral vasculitis $(17,18)$. Herein we report 3 cases of peripheral gangrene as the presentation of $\mathrm{KD}$.

\section{Case Presentation}

\subsection{Case 1}

A 4-month-old boy presented with prolonged fever from since a month ago. Although sepsis work up and wide-spectrum anti-biotic therapy were done, there was no recovery. He was ill, febrile and severely anemic. In physical examination, pallor, hepato-splenomegaly and $3 / 6$ systolic murmur were detected. He had peripheral gangrene on both sides lower and right side upper extremities. Peripheral pulses were undetectable. Lab data were as follow: WBC $14.1 \times 10^{3} / \mathrm{mm}^{3}$ (Neut $61 \%$ ), $\mathrm{Hb} 7.1 \mathrm{~g} / \mathrm{dl}$, Plt $329 \times 10^{3} \mu$ L, CRP 201, ESR 74, Ammonia 1.2, Lactate $=27$, negative cultures, sterile pyuria and normal electrolytes except hyponatremia (Na 129). Anti-phospholipid (apl) antibodies and anti-neutrophil cytoplasmic antibodies (ANCAs) were negative. Gallbladder wall thickness and edema with no hydrops, hepatomegaly and splenomegaly were detected in the abdominal sonography. Doppler sonography showed proximal stenosis in right side brachial artery and distal arteries of both lower extremities. Bone marrow aspiration was normal. Multiple aneurysms in the left coronary artery and other aortic branches were seen in the echocardiography, so magnetic resonance arteriog-

Copyright (C) 2015, Growth \& Development Research Center. This is an open-access article distributed under the terms of the Creative Commons Attribution-NonCommercial 4.0 International License (http://creativecommons.org/licenses/by-nc/4.0/) which permits copy and redistribute the material just in noncommercial usages, provided the original work is properly cited. 
Malekzadeh I et al.

raphy was done which showed multiple aneurysms in all coronary branches, both subclavian, external and internal iliac, bronchial, renal and mesenteric arteries (Figure 1). Because of KD as the diagnosis, treatment with high dose aspirin, methylprednisolone pulse therapy and IVIG initiated. Anti-coagulant therapy with heparin was done for peripheral gangrene and multiple arterial stenosis. Due to multiple aneurysms cyclophosphamide was added. The arterial dilations, aneurysms and organomegalies slightly improved. Unfortunately, peripheral gangrene remained and auto-amputation happened in distal phalanxes of the right hand and left foot (Figure 2). Follow-up of the patient after 1 year showed very mild improvement in the size of coronary aneurysms, but peripheral aneurysms were without improvement.

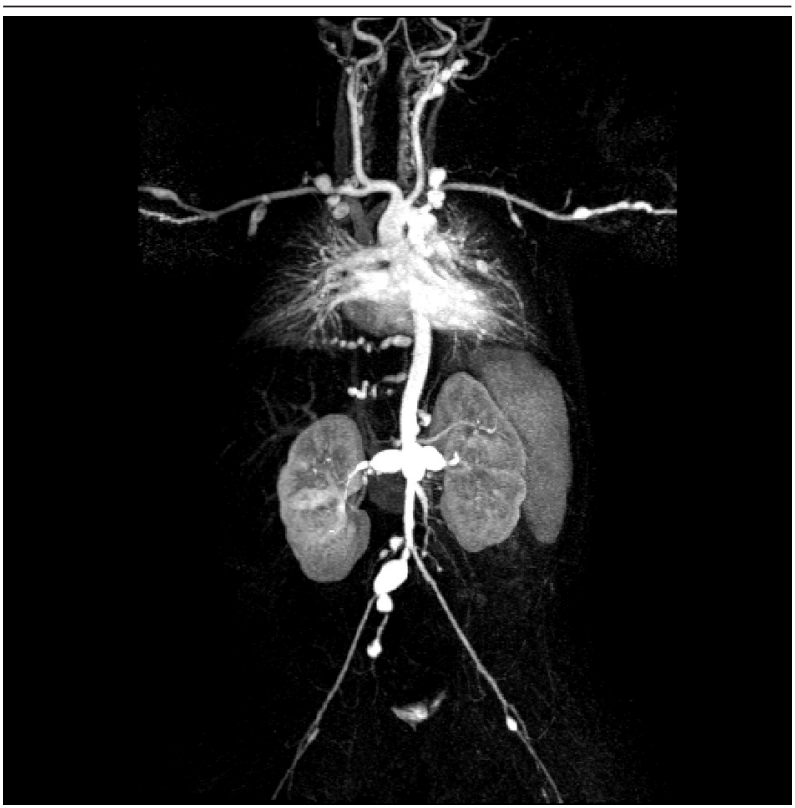

Figure 1. Multiple Aneurysms in Coronary and Systemic Arteries

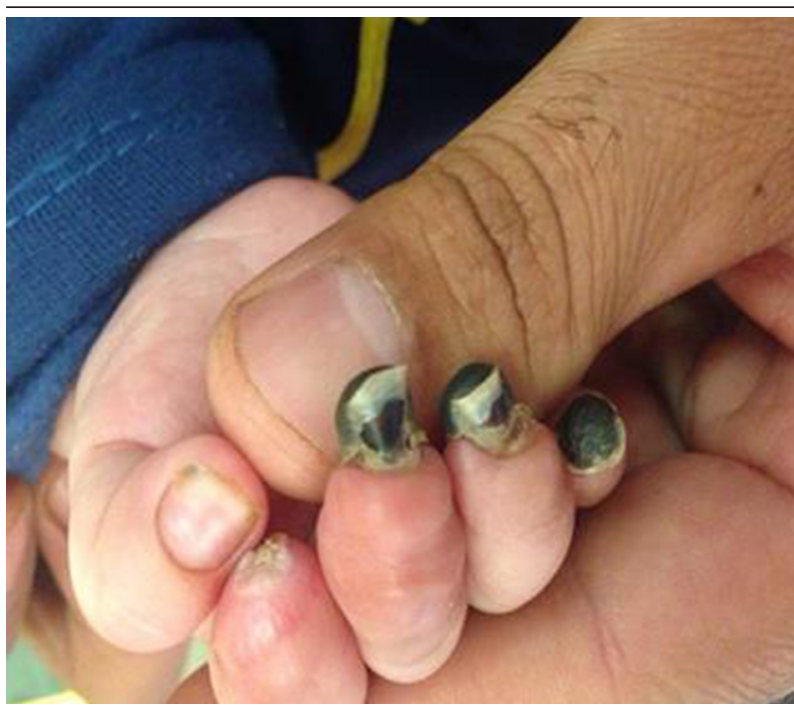

Figure 2. Peripheral Gangrene as a Presentation of Kawasaki Disease

\subsection{Case 2}

Six-month-old girl, a known case of PHACE (Posterior fossa abnormalities, Hemangioma, Arterial lesions, Cardiac abnormalities/Aortic coarctation, and Eye abnormalities) syndrome was referred with 2-days history of fever, irritability and poor feeding. She had coryza and cough. At admission, she was febrile and irritable. Cardiac auscultation was abnormal due to aortic coarctation, but lung sounds were normal. There were no organomegalies. But the most important sign was distal cyanosis and scaling in hands and gangrene of the middle phalanx of the right hand, finger 3. Lab tests were normal: WBC $13.4 \times 10^{3} / \mathrm{mm}^{3}$ (Neut 45.1\%), Hb $9.5 \mathrm{~g} / \mathrm{dl}$, Plt $470 \times 10^{3} \mu \mathrm{L}$, CRP 0.5, ESR 9, negative cultures and sterile pyuria. Pleocytosis $\left(22.77 \times 10^{3} / \mathrm{mm}^{3}\right)$, thrombocytosis $\left(705 \times 10^{3} \mu \mathrm{L}\right)$ and ESR rising (57) happened, during hospitalization. Apl antibodies and ANCAs were negative. Abdominal sonography and chest X-ray were normal. Due to dilation of descending aorta in echocardiography and angiography in addition to thrombocytosis and peripheral gangrene, Kawasaki disease was diagnosed. She was treated with aspirin, methylprednisolone, and IVIG, which terminated the fever. Peripheral cyanosis and gangrene bettered. In follow up after 6 months, dilatation of coronary improved relatively.

\subsection{Case 3}

A 1-year-old boy was referred with high grade fever and intensifying peripheral gangrene. He was pretty ill, febrile and irritable. First he was admitted with a history of fever for 2 weeks, therefore sepsis work up and antibiotic therapy initiated. Sucessively strawberry tongue, cervical lymphadenopathy and scaling appeared. Due to probability of Kawasaki disease, echocardiography was done which showed mild pericardial effusion and normal coronary arteries. Then he was treated with IVIG and aspirin. During the hospital stay, swelling and erythema of extremities happened. At the 6th day of admission, peripheral cyanosis and gangrene in distal phalanx of extremities happened. Because of continuous fever and peripheral gangrene, he was referred to our tertiary center (Figure 3). In second echocardiography, in addition to pericardial effusion, multiple aneurysms in RCA, LMCA, and LAD were detected. He had leukocytosis (32.73 $\left.\times 10^{3} / \mathrm{mm}^{3}\right)$, anemia $(\mathrm{Hb} 10 \mathrm{~g} / \mathrm{dl})$, and severe thrombocytosis $\left(1.344 \times 10^{3} \mu \mathrm{L}\right)$. In addition to aspirin and IVIG, methylprednisolone, azathioprine and heparin as an anti-coagulant were prescribed. Fever period ended and peripheral gangrene got better. Echocardiography after pulse of methylprednisolone showed decrease in size of aneurysms and no pericardial effusion. FANA level was 1/80 in speckled format, anti dsDNA level was 128 (normal range $<100$ ) and complementary system evaluation was normal. In the acute phase of disease ANCAs and apl antibodies were negative. After 2 months, FANA and anti dsDNA became normal but there was no recovery in coro- 
nary aneurysms. He was admitted again. This time echocardiography findings were as follow: LMCA diameter 7.4 $\mathrm{mm}$, RCA $4.3 \mathrm{~mm}$, and LAD $5.2 \mathrm{~mm}$. With continuation of aspirin, warfarin, and azathioprine (for 3 months), second pulse of methylprednisolone and IVIG and Infliximab were given. After a year cardiovascular angiography revealed: $4.5 \mathrm{~mm}$ diameter aneurysm in distal LMCA, LAD stenosis and non-appropriate tapering of RCA. His treatment continued with long-term use of low dose aspirin and warfarin for prevention of thrombosis.

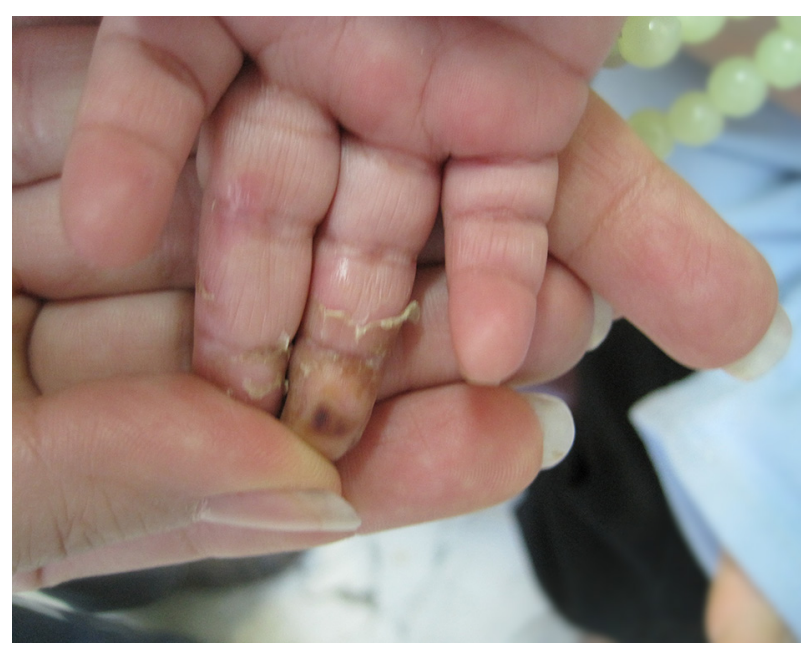

Figure 3. Distal Cyanosis and Scaling in Hands and Gangrene of the Distal Phalanx of the Middle Finger in the Repair Phase

\section{Discussion}

Gangrene is a rare presentation of Kawasaki disease that needs a high level of suspicion by physicians to discover the underlying disease. There are reports of peripheral gangrene due to KD from around the world, however it seems that the reports are less from Japan as a country from where most of the KD cases are reported (17). There is not a clear explanation why gangrene happens in $\mathrm{KD}$, but some reasons have been emphasized as etiology such as arteritis, arteriospasm, thrombosis of inflamed vessels and decreased peripheral perfusion (17).

Most of the reported cases of gangrene due to $\mathrm{KD}$, experienced a degree of sequel especially amputation of fingers, this has also occurred in our cases. However there is a report with no sequel from a 57 day infant in Korea in whom IVIG, dexamethasone, methotrexate and anticoagulant were administered (19). There is a wide range of different medications which are used in management of KD with gangrene. Some of the treatment agents that have been used are heparin, steroids, hydralazine, propranolol, warfarin, urokinase, PGE1, dipyridamole, nitroprusside, tissue plasminogen activator, prostacyclin, glyceryl trinitrate, nifedipin, and even caudal block (20). We additionally used cyclophosphamide due to multiple aneurysms in our first case and infliximab in case 3.

Regarding age of this complication, most of the reports present young age in infancy especially under seven months of age (20). But it is not a real clear cut for age as we have reported a one year old child with gangrene. There is also a report of a 22 year old man with Kawasaki whose disease was complicated by gangrene and amputation of fingers (21).

We reported three cases of peripheral gangrene to alert the physicians about this rare and important complication of KD, because sometimes this condition of a patient may be the first presentation of KD such as the infant we reported as our first case. Therefore, without a single guideline for managing peripheral gangrene of $\mathrm{KD}$, more cases should be reported to understand the epidemiology of the disease and experimental studies should be done to evaluate the best medications for treatment of gangrene complicated KD.

Peripheral gangrene must be seen as an important sign of infantile Kawasaki disease. Early aggressive treatment after IVIG therapy with methylprednisolone pulse therapy and/or cytotoxic or biologic drugs can prevent permanent morbidities and sequels.

\section{References}

1. Son MBF, Newburger JW. Kawasaki Disease. Nelson Textbook of Pediatrics. 20th ed. Philadelphia: Elsevier; 2016. pp.1209-14.

2. Makino N, Nakamura Y, Yashiro M, Ae R, Tsuboi S, Aoyama Y, et al. Descriptive epidemiology of Kawasaki disease in Japan, 2011-2012: from the results of the 22nd nationwide survey. J Epidemiol. 2015;25(3):239-45. doi: 10.2188/jea.JE20140089. [PubMed: 25716368]

3. Holman RC, Curns AT, Belay ED, Steiner CA, Effler PV, Yorita $\mathrm{KL}$, et al. Kawasaki syndrome in Hawaii. Pediatr Infect Dis J. 2005;24(5):429-33. [PubMed: 15876942]

4. Burns JC, Kushner HI, Bastian JF, Shike H, Shimizu C, Matsubara T, et al. Kawasaki disease: A brief history. Pediatrics. 2000;106(2):E27. [PubMed:10920183]

5. Kato H, Sugimura T, Akagi T, Sato N, Hashino K, Maeno Y, et al. Long-term consequences of Kawasaki disease. A 10- to 21-year follow-up study of 594 patients. Circulation. 1996;94(6):1379-85. [PubMed: 8822996]

6. Newburger JW, Sanders SP, Burns JC, Parness IA, Beiser AS, Colan SD. Left ventricular contractility and function in Kawasaki syndrome. Effect of intravenous gamma-globulin. Circulation. 1989;79(6):1237-46. [PubMed: 2720925]

7. No SJ, Kim DO, Choi KM, Eun LY. Do predictors of incomplete Kawasaki disease exist for infants? Pediatr Cardiol. 2013;34(2):28690. doi:10.1007/s00246-012-0440-3. [PubMed: 23001516]

8. Chang RK. Hospitalizations for Kawasaki disease among children in the United States, 1988-1997. Pediatrics. 2002;109(6):e87. [PubMed: 12042581]

9. Moradinejad MH, Kiani A. Kawasaki disease in 159 iranian children. Iran J Pediatrics. 2007;17(3):241-6.

10. Takahashi M, Mason W, Lewis AB. Regression of coronary an eurysms in patients with Kawasaki syndrome. Circulation. 1987;75(2):387-94. [PubMed:3802442]

11. Pannaraj PS, Turner CL, Bastian JF, Burns JC. Failure to diagnose Kawasaki disease at the extremes of the pediatric age range. Pediatr Infect Dis J. 2004;23(8):789-91. [PubMed: 15295237]

12. Shprakh VV, Surikova ZV, Bregel LV, Subbotin VM. [A case of the cerebral vasculitis at the late stage of Kawasaki disease]. $Z \mathrm{~h}$ Nevrol Psikhiatr Im S S Korsakova. 2014;114(11):107-10. [PubMed: 25591646]

13. Hematian MN, Torabi S, MalaKan-Rad E, Sayadpour-Zanjani K, 


\section{Malekzadeh I et al.}

Ziaee V, Lotfi-Tolkaldany M. Noninvasive Evaluation of Myocardial Systolic Dysfunction in the Early Stage of Kawasaki Disease: A Speckle-Tracking Echocardiography Study. Iran J Pediatr. 2015;25(3):e198. doi:10.5812/ijp.25(3)2015.198. [PubMed: 26199701]

14. Sahoo S, Mandal AK. Congestive heart failure - an atypical presentation of kawasaki disease. Iran J Pediatr. 2012;22(3):428-9. [PubMed: 23399870]

15. Pires A, Sousa G, Castela E. Coronary and systemic aneurysms in an infant with Kawasaki disease. Pediatr Cardiol. 2009;30(4):5689. doi:10.1007/s00246-009-9429-y. [PubMed:19365659]

16. Heran MK, Hockley A. Multiple mirror-image peripheral arterial aneurysms in Kawasaki disease. Pediatr Cardiol. 2011;32(5):670-3. doi:10.1007/s00246-011-9916-9. [PubMed: 21327891]

17. Tomita S, Chung K, Mas M, Gidding S, Shulman ST. Periphera gangrene associated with Kawasaki disease. Clin Infect Dis
1992:14(1):121-6. [PubMed:1571415]

18. Durall AL, Phillips JR, Weisse ME, Mullett CJ. Infantile Kawasaki disease and peripheral gangrene. J Pediatr. 2006;149(1):131-3. doi: 10.1016/j.jpeds.2006.02.043. [PubMed:16860142]

19. Kim NY, Choi DY, Jung MJ, Jeon IS. A case of refractory Kawasaki disease complicated by peripheral ischemia. Pediatr Cardiol. 2008;29(6):1110-4. doi:10.1007/s00246-008-9221-4. [PubMed: 18481137]

20. Chang JS, Lin JS, Peng CT, Tsai CH. Kawasaki disease complicated by peripheral gangrene. Pediatr Cardiol. 1999;20(2):139-42. doi: 10.1007/s002469900422. [PubMed: 9986892]

21. Bonte I, Mahr A, Laroche L, Guillevin L, Robineau M. Periphera gangrene in adult-onset Kawasaki disease. Scand J Rheumatol. 2005;34(1):71-3. [PubMed:15903031] 